Review Article

\title{
Intervention Mechanisms of Xinmailong Injection, a Periplaneta Americana Extract, on Cardiovascular Disease: A Systematic Review of Basic Researches
}

\author{
Shan-Shan Lin $\left(\mathbb{D},{ }^{1}\right.$ Chun-Xiang Liu $\mathbb{D},{ }^{2}$ Xian-Liang Wang $\left(\mathbb{D},{ }^{1}\right.$ and Jing-Yuan Mao ${ }^{1}{ }^{1}$ \\ ${ }^{1}$ Cardiovascular Department, First Teaching Hospital of Tianjin University of Traditional Chinese Medicine, Tianjin 300381, China \\ ${ }^{2}$ Evidence-Based Medicine Center, Tianjin University of Traditional Chinese Medicine, Tianjin 301617, China \\ Correspondence should be addressed to Xian-Liang Wang; xlwang1981@126.com and Jing-Yuan Mao; jymao@126.com
}

Received 12 April 2019; Accepted 25 June 2019; Published 22 July 2019

Guest Editor: Irawan W. Kusuma

Copyright (c) 2019 Shan-Shan Lin et al. This is an open access article distributed under the Creative Commons Attribution License, which permits unrestricted use, distribution, and reproduction in any medium, provided the original work is properly cited.

Background. At present, the prevention and treatment of cardiovascular disease in the world are facing severe challenges. Xinmailong injection, which is derived from the animal medicine Periplaneta Americana, has certain advantages in the clinical treatment of cardiovascular disease. This study systematically evaluated the basic research reports of Xinmailong Injection on cardiovascular disease and made its pharmacological mechanisms more clear. Methods. Basic research reports on the intervention mechanisms of Xinmailong Injection on cardiovascular disease in PubMed, EMBASE, Cochrane Library (No. 2, 2019), CNKI, Wan Fang, and VIP databases were searched. The search time limit was from the establishment of the database to February 2019. The literature was screened according to inclusion and exclusion criteria, and then the data were extracted and a descriptive analysis of the pharmacological mechanisms of Xinmailong Injection on cardiovascular disease was performed. Results. Finally, twenty-two basic research reports were included. The intervention mechanisms of Xinmailong Injection on cardiovascular disease mainly includes the following: inhibiting oxidative stress and inflammatory reaction; regulating autophagy; promoting $\mathrm{Ca}^{2+}$ influx by activating excitability of excitation-contraction coupling (ECC); inhibiting overexpressions of transforming growth factor- $\beta 1$ (TGF- $\beta 1$ ) and connective tissue growth factor (CTGF) to regulate the dynamic balance of matrix metalloproteinases (MMPs) and tissue inhibitors of matrix metalloproteinases (TIMPs); inhibiting the phosphorylation of extracellular regulated protein kinases $1 / 2(\mathrm{ERK} 1 / 2)$, protein kinase B (AKT), and glycogen synthase kinase $3 \beta(\mathrm{GSK} 3 \beta)$ proteins and overexpression of the downstream transcription factor GATA4 in the nucleus; regulating vascular endothelial factors and so on. Conclusions. Xinmailong Injection can protect cardiomyocytes and maintain the normal function of the heart in various ways, thus effectively preventing the development of cardiovascular disease. Therefore, Xinmailong Injection has great potential for clinical application, and more basic researches need to be carried out to explore the medicinal value of Xinmailong Injection.

\section{Background}

Cardiovascular disease is currently the first cause of death in the world. According to the "China Cardiovascular Disease Report 2017" [1], the number of people suffering from cardiovascular disease in China is about 290 million. At present, the prevention and treatment of cardiovascular disease have achieved initial results, but they still face serious challenges. Animal material medicine is an important part of traditional medicinal resources. A large number of animal material medicine have been developed as important raw materials for modern Chinese patent medicines and western medicines due to their special biological activities. Periplaneta Americana is part of them. Due to its rich source and low cost, the medicinal value of $P$. Americana has enormous development potential. Since the 1980s, Chinese pharmacologist Professor Li Shunan has developed a series of new drugs, such as Kangfuxin Liquid, Xinmailong Injection, and Ganlong Capsule, using the P. Americana as raw material. They are used in many fields such as fighting tumors, improving heart function, inhibiting liver fibrosis, and protecting the gastrointestinal mucosa [2]. Among them, 
Xinmailong Injection is mainly an auxiliary drug for chronic heart failure $(\mathrm{CHF})$, and its main active ingredients are adenosine, inosine, protocatechuic acid, and pyroglutamate dipeptides [3-5]. Xinmailong Injection became the second class of traditional Chinese medicines in the national new drugs in 2006, which was approved by the State Food and Drug Administration (SFDA). Its efficacy in treating cardiovascular disease has been highly recognized since its launch. It has been recommended by many expert consensus and guidelines such as "Expert Consensus on Diagnosis and Treatment of Chronic Heart Failure by Integrative Chinese and Western Medicine" [4] and "Guidelines for the Diagnosis and Treatment of Acute Myocardial Infarction by Integrative Chinese and Western Medicine" [6].

In this study, the basic researches about the intervention mechanisms of Xinmailong Injection on cardiovascular disease were systematically organized. The aim is to provide a reference for further research and clinical application of Xinmailong Injection.

\section{Methods}

2.1. Inclusion Criteria. The inclusion criteria are the basic researches about the intervention mechanisms of Xinmailong Injection on cardiovascular disease. Experimental models include animals, organs, tissues, and cells associated with cardiovascular disease.

2.2. Exclusion Criteria. Exclusion criteria were (1) to observe the comprehensive efficacy of Xinmailong Injection combined with other interventions; (2) for the repeated publication of the literature, excluding low-quality literature; (3) the full text not obtained; (4) clinical research, case reports, expert consensus, review, systematic review, and meta-analysis.

2.3. Search Strategy. The basic researches about the intervention mechanisms of Xinmailong Injection on cardiovascular disease in PubMed, EMBASE, Cochrane Library (No. 2 of 2019), CNKI, Wan Fang, and VIP databases were searched. The search time was from the database establishment to February 2019. Search terms included P. Americana, American cockroach, Xinmailong, heart failure, ventricular dysfunction, blood vessel, artery, heart, cardiac, cardiovascular, myocardium, myocardial, and the like. The search strategy was a combination of subject words and free words. In addition, we screened the reference list of relevant literature to avoid omissions. Take PubMed as an example. The specific search strategy is shown in Table 1.

2.4. Literature Screening and Data Extraction. Literature screening and data extraction were performed according to the inclusion and exclusion criteria. The data extraction includes title, author, publication year, experimental model, observation indicators, and research result, etc. Considering this article is a summary of the intervention mechanisms of Xinmailong Injection on cardiovascular disease, a descriptive analysis was performed.

\section{Results}

3.1. Literature Screening Process and Results. A total of 1135 pieces of literature were retrieved and 46 pieces of possible related literature were screened out. After reading the full text, twenty-two pieces of literature were finally included. The literature screening process and results are shown in Figure 1.

3.2. Literature Overview. The included literature consists of 4 pieces of English literature and 18 Chinese literature. The literature was published in 1995 2019. The literature published in the past five years accounted for $45.45 \%(10 / 22)$. Experimental models included animals with cardiovascular disease (such as myocardial cell injury, myocardial ischemiareperfusion injury, myocardial infarction, atherosclerosis, cardiomyopathy, heart failure, or pulmonary hypertension, etc.), isolated rat heart, $\mathrm{H} 9 \mathrm{C} 2$ cells, and hypoxicreoxygenated rat cardiomyocytes, and the like. See Table 2 for details.

3.3. Intervention Mechanism of Xinmailong Injection on Cardiovascular Disease. The main indication for Xinmailong Injection is CHF, which is the terminal stage of the progressive development of various cardiovascular diseases. The early manifestations of heart failure (HF) are myocardial cell damage, hypertrophy, and necrosis, followed by myocardial fibrosis (MF) and ventricular enlargement. Therefore, Xinmailong Injection can also be widely used in the treatment of other cardiovascular diseases. Current researches show that Xinmailong Injection mainly interferes with the cardiovascular system through the following various mechanisms.

3.3.1. Protective Mechanism of Xinmailong Injection on Cardiomyocytes. Studies have shown that myocardial cell damage is closely related to Oxidative Stress (OS) and inflammation [7]. OS refers to an unbalanced state caused by excessive oxidation of the body. In this state, the body's ability to synthesize reactive oxygen species (ROS) increases and the ability to resist oxidation decreases, resulting in damage to the body's tissue function. When OS occurs, the levels of lipid peroxides and ROS are significantly increased. The increase of ROS levels can further increase the expression of proinflammatory factors by activating nuclear factor kappa $\mathrm{B}$ (NF-KB) system, thereby promoting the inflammatory response $[8,9]$.

$\mathrm{Wu}$ Jianxin et al. [10] found that Xinmailong Injection can significantly reduce the J-point displacement (increased or lowered) on the electrocardiogram (ECG) of rabbits caused by isoproterenol so that the J-point position is close to the normal equipotential line. This suggests that Xinmailong Injection has a protective effect on isoproterenol-induced myocardial ischemic injury. Xinmailong Injection can also significantly reduce the frequency of ischemic arrhythmias in rabbits. Studies [11] on the rats further confirmed that Xinmailong Injection can correct the J-point displacement on the ECG caused by myocardial ischemia, and Xinmailong Injection can significantly reduce the level of the lipid peroxide malondialdehyde (MDA), reduce the activity of 


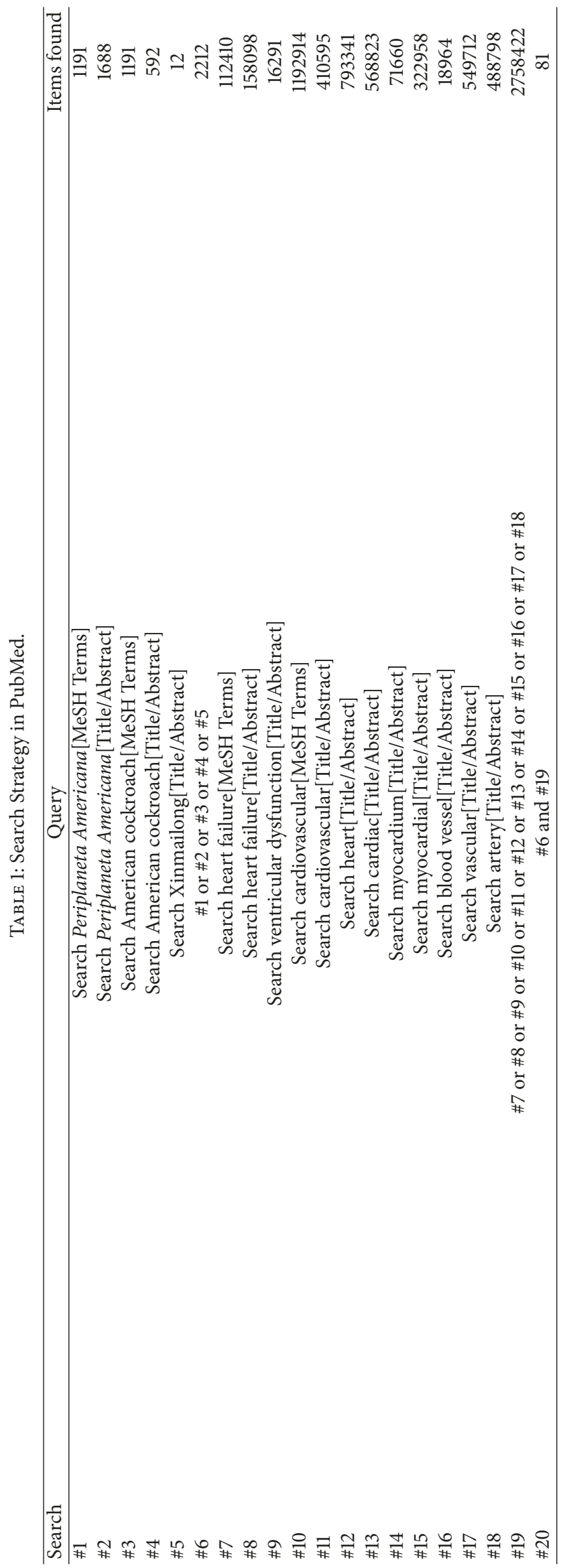




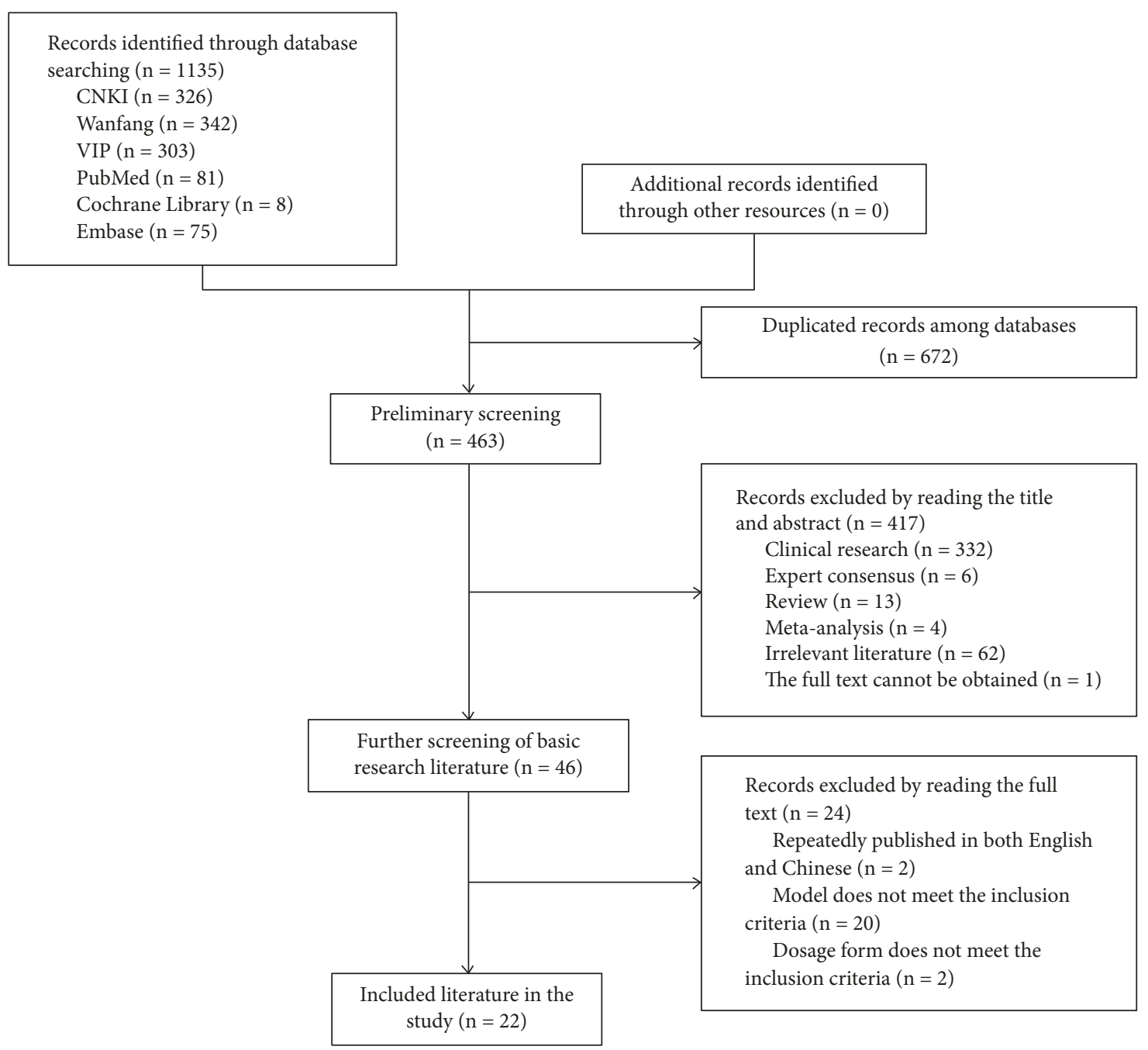

FIGURE 1: Flowchart of the literature selection process.

creatine phosphokinase $(\mathrm{CPK})$ and lactate dehydrogenase $(\mathrm{LDH})$ in blood, and improve glutathione peroxidase (GSHPX) and SOD activity in myocardial tissue. Tian Kunlun and Jiang $\mathrm{Yu}$ [12] found that Xinmailong Injection can increase SOD activity and $\mathrm{NO}_{2}{ }^{-} / \mathrm{NO}_{3}{ }^{-}$(ratio of nitrite to nitrate) level, correct acidosis, improve circulating blood flow, and increase urine output in rabbits of ischemiareperfusion injury induced by hypovolemic shock. Li Zhengtao et al. [13] found in the cell experiment that Xinmailong Injection can significantly reduce the production of ROS in $\mathrm{H} 9 \mathrm{C} 2$ cells and increase the expression of antioxidant enzymes such as superoxide dismutase- (SOD-) 1, SOD2 , and heme oxygenase ( $\mathrm{HO})-1$, which indicates that the protective effect of Xinmailong Injection on cardiomyocytes is achieved by anti-lipid peroxidation and inhibition of OS.

By observing the protective effect of Xinmailong Injection on myocardial ischemia-reperfusion injury in young rabbits, Cao Hongxiao et al. [14] found that Xinmailong Injection can significantly inhibit the levels of endothelial constitutive nitric oxide synthase (ecNOS), MDA, and creatine kinase (CK) and the infiltration of inflammatory cells after reperfusion. Therefore, the researchers believe that Xinmailong Injection reduces the production of oxygen free radicals by anti-lipid peroxidation, which inhibits the inflammatory response, thereby reducing the damage of vascular endothelial cells and cardiomyocytes caused by reperfusion. Zhang Lijuan et al. [15] found that the levels of serum CK-MB, myocardial NF- $\kappa \mathrm{B}$, and TNF- $\alpha$ reached a peak in neonatal rats after 6 hours of asphyxia. Moreover, the expression of NF$\kappa \mathrm{B}$ and TNF- $\alpha$ was positively correlated $(\mathrm{r}=0.979, \mathrm{P}<0.01)$. Xinmailong Injection can significantly inhibit the above changes. This suggests that Xinmailong Injection can reduce the expression of proinflammatory factors by inhibiting the activation of the NF-kB system, thereby protecting the myocardial injury. Xinmailong Injection can significantly inhibit the above myocardial damage. Combined with previous studies, it can be inferred that Xinmailong Injection can reduce the expression of proinflammatory factors by inhibiting the activation of the NF-kB system. 


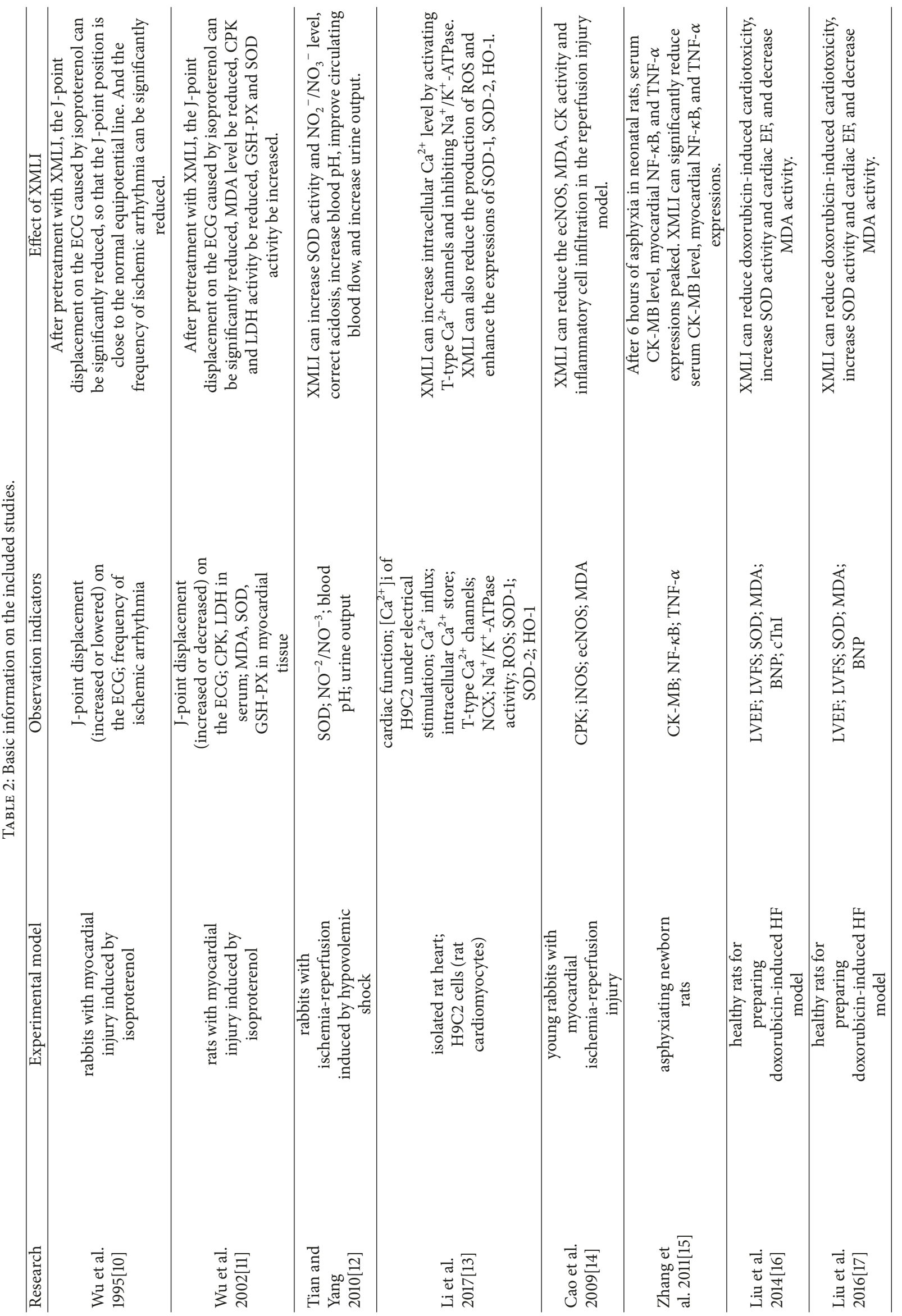




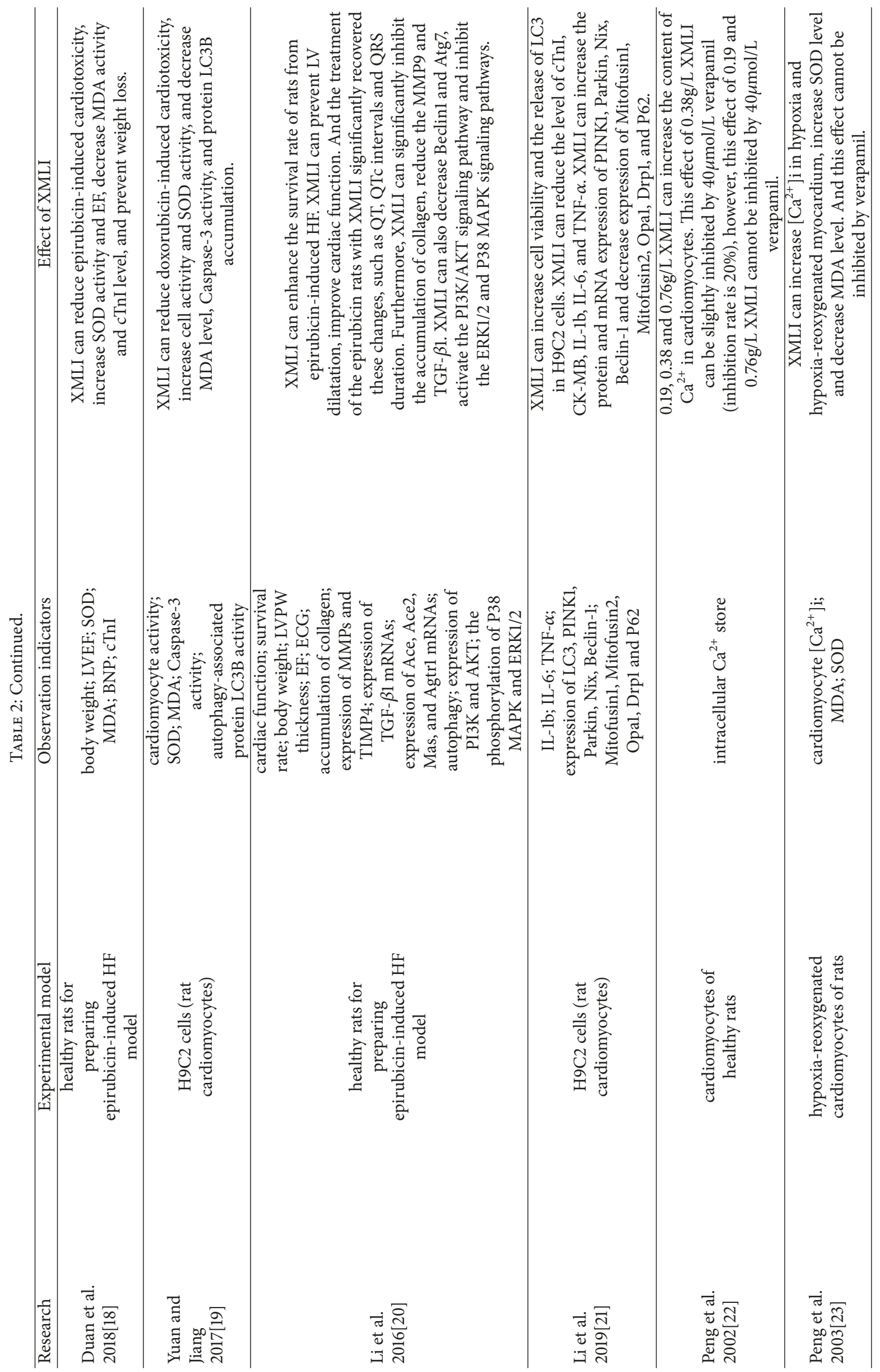




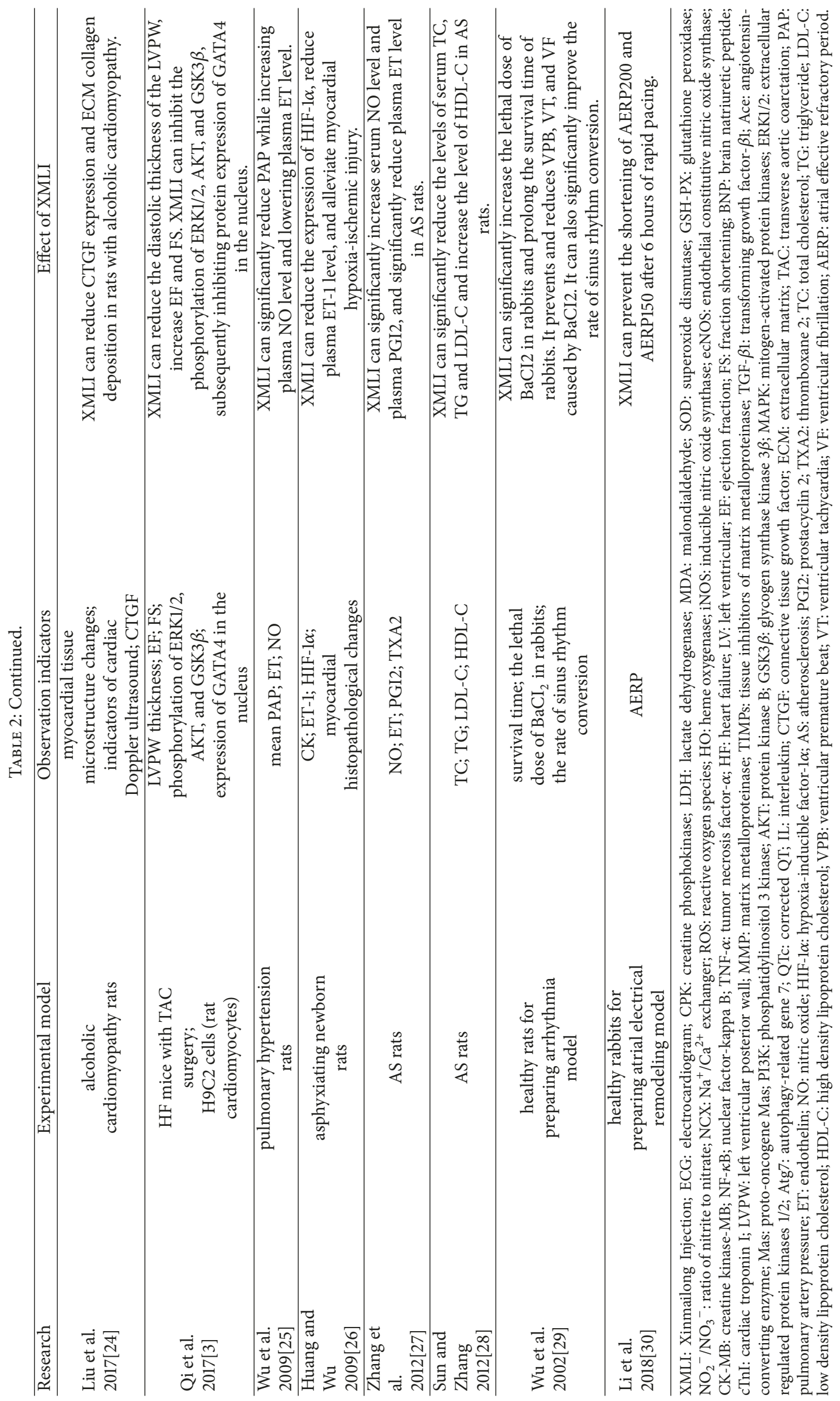


3.3.2. Defensive Mechanism of Xinmailong Injection on Drug Cardiotoxicity. Autophagy refers to the process by which cells use lysosomes to degrade their damaged organelles and macromolecules. This process is regulated by the autophagyrelated gene (Atg). The signaling pathways that regulate autophagy are complex. These signaling pathways interact with each other and form a huge regulatory network. Studies have shown that ROS is one of the major intracellular signal transducers that maintain autophagy [31]. At the same time, autophagy, as a protective and defense mechanism widely present in cells, plays an important role in alleviating ROSmediated cell damage [32].

Liu Wei et al. [16] found that long-term use of doxorubicin significantly reduced the SOD activity and increased the MDA level in rats. While taking doxorubicin, loading and using Xinmailong Injection can increase the SOD activity and MDA level slightly. The results indicate that Xinmailong Injection can alleviate the cardiotoxicity of anthracyclines by activating the endogenous ROS scavenging system. Xinmailong Injection has the same preventive effect on myocardial toxicity induced by doxorubicin and epirubicin $[17,18]$. Yuan Lili and Yang Zhihua [19] observed that the activity of H9C2 cells induced by doxorubicin was significantly decreased, the level of SOD in cells decreased and the level of MDA increased, the activity and expression of Caspase- 3 in cells increased, and the accumulation of autophagy-related protein LC3B increased. The Xinmailong Injection can obviously reduce the cardiotoxicity induced by doxorubicin. This study suggests that the protective effect of Xinmailong Injection is related to the regulation of autophagy.

Li Hui et al. [20] conducted the autophagy-related studies and found that the increase in PI3K/AKT levels and the inhibition of P38MAPK and ERK1/2 phosphorylation contribute to the enhancement of anti-autophagy activity of Xinmailong Injection. Therefore, the researchers believed that Xinmailong Injection can inhibit autophagy by activating PI3K/AKT signaling pathway and inhibiting ERK1/2 and P38 MAPK signaling pathways, which is an important mechanism for Xinmailong Injection to reduce the cardiotoxicity of drugs.

Li Jie et al. [21] found that Xinmailong Injection significantly inhibited the upregulation of interleukin- (IL-) $1 \mathrm{~b}$, IL-6, and tumor necrosis factor- (TNF-) $\alpha$ levels and the decrease of LC3 release in H9C2 cells. However, this effect was inhibited by mitochondrial autophagy inhibitor Mdivi1 and autophagy-related protein Atg7 siRNA. The study also found that Xinmailong Injection significantly inhibited the expression of PINK1, Parkin, Nix, and Beclin-1 and promoted the expression of Mitofusin1, Mitofusin2, Opal, Drp1, and P62. This suggests that Xinmailong Injection can reduce the cardiotoxicity of the drug by regulating autophagy mediated by the PINK1/Parkin signaling pathway.

3.3.3. Mechanism of Xinmailong Injection Enhancing Myocardial Contractility. Studies have shown that the enhancement of myocardial contractility can be achieved by activating myocardial excitation-contraction coupling (ECC) excitability to promote $\mathrm{Ca}^{2+}$ influx in cardiomyocytes. ECC refers to the signal transduction process of cell membrane depolarization mediated by $\mathrm{Ca}^{2+}$. When ECC excitability is activated, the $\mathrm{Ca}^{2+}$ on the cell membrane is transiently increased. At this time, $\mathrm{Ca}^{2+}$ and troponin are combined, and the transverse bridge on the thick muscle wire is combined with the thin filament to oscillate, thereby causing the myocardial cells to contract. The transport stability of $\mathrm{Ca}^{2+}$ is achieved by adjusting the voltage-dependent $\mathrm{Ca}^{2+}$ channel, $\mathrm{Ca}^{2+}$-ATPase, and $\mathrm{Na}^{+} / \mathrm{Ca}^{2+}$ exchanger.

Peng Fang et al. [22, 23] conducted multiple studies and found that Xinmailong Injection can promote $\mathrm{Ca}^{2+}$ influx in rat cardiomyocytes, and this effect cannot be inhibited by verapamil, a $\mathrm{Ca}^{2+}$ channel blocker. Therefore, it is concluded that Xinmailong Injection does not promote $\mathrm{Ca}^{2+}$ influx through $\mathrm{Ca}^{2+}$ channels. However, a further study [13] by Li Zhengtao et al. using $\mathrm{Ca}^{2+}$ imaging technology in $\mathrm{H} 9 \mathrm{C} 2$ cells revealed that Xinmailong Injection can be inhibited by ML218- $\mathrm{HCl}$ (a T-type $\mathrm{Ca}^{2+}$ channels antagonist instead of Ltype $\mathrm{Ca}^{2+}$ channel). Therefore, it is believed that Xinmailong Injection can increase cellular $\mathrm{Ca}^{2+}$ influx by activating $\mathrm{T}$ type $\mathrm{Ca}^{2+}$ channels. Meanwhile, Li Zhengtao et al. also observed that Xinmailong Injection can inhibit $\mathrm{Na}^{+} / \mathrm{K}^{+}$adenosine triphosphate (ATP) enzyme activity. This process can reduce the transmembrane electrochemical gradient of $\mathrm{Na}^{+}$, weaken the $\mathrm{Na}^{+} / \mathrm{Ca}^{2+}$ exchange capacity, reduce the $\mathrm{Ca}^{2+}$ export, and increase the concentration of $\mathrm{Ca}^{2+}$ in the myocardial cells, thereby enhancing the myocardial contractility.

3.3.4. Mechanism of Xinmailong Injection Inhibiting MF. MF is a pathological phenomenon in which cardiac fibroblasts (CFs) abnormally proliferate and collagen deposition in the extracellular matrix (ECM), imbalance of various types of collagen, and disordered collagen arrangement are observed [33]. This process is one of the main causes of ventricular remodeling and cardiac ejection disorders $[34,35]$.

Transforming growth factor- $\beta$ (TGF- $\beta$ ) is an important cytokine during MF [36]. It promotes the differentiation of resting fibroblasts into CFs. CFs can synthesize and secrete large amounts of collagen and can also produce matrix metalloproteinases (MMPs) and tissue inhibitors of matrix metalloproteinase (TIMPs) [37]. MMPs are a group of $\mathrm{Zn}^{2-}$ dependent proteolytic enzymes that specifically and efficiently degrade ECM. TIMPs can maintain the normal structure and function of ECM by regulating the activity of MMPs. Under physiological conditions, the expression of MMPs was extremely low; under pathological conditions, MMPs expression was significantly upregulated. Studies have shown that the occurrence of MF is related to the upregulation of MMP-2 and MMP-9 expression [38]. Li Hui et al. [20] found that Xinmailong Injection can reduce the levels of TGF- $\beta 1$ mRNA and MMP-9 in the serum of rats with $\mathrm{HF}$. Clinical studies have also confirmed that Xinmailong Injection can inhibit the upregulation of MMP1 and MMP-9 expression in serum of $\mathrm{HF}$ patients $[39,40]$ and reduce ECM collagen deposition, thereby counteracting MF. 
Connective tissue growth factor (CTGF) is a downstream factor specifically induced by TGF- $\beta$. It mediates the profibrotic progression of TGF- $\beta$ and is an excellent marker of MF. The expression of TGF- $\beta$ and CTGF increased synchronously during fibrosis, which together promoted ECM collagen deposition [41, 42]. Liu Guohong et al. [24] found that Xinmailong Injection can significantly inhibit the expression of CTGF and decrease the deposition of ECM collagen in cardiomyocytes of rats with alcoholic cardiomyopathy, indicating that Xinmailong Injection can effectively delay the process of MF.

3.3.5. Mechanism of Xinmailong Injection Delaying the Development of Cardiac Hypertrophy. Cardiac hypertrophy is a complex dynamic process that is regulated by multiple factors. Its early change is an adaptive compensatory response. This reaction is caused by pathological factors such as excessive pressure or volume overload. With the longterm existence of pathological stimuli, compensatory cardiac hypertrophy may eventually develop into CHF.

Qi Jianyong et al. [3] found that Xinmailong Injection can effectively improve cardiac structure and function abnormalities in mice with transverse aortic coarctation (TAC) surgery caused by pressure overload and reduce ejection fraction and the thickness of the diastolic left ventricular posterior wall (LVPW). The downstream transcription factor GATA4 is a nuclear transcription factor closely related to cardiac development and plays a key role in the development of cardiomyocyte differentiation, cardiac hypertrophy, and HF [43]. Qi Jianyong et al. further carried out experiments in $\mathrm{H} 9 \mathrm{C} 2$ cells and found that Xinmailong Injection can inhibit the phosphorylation of ERK1/2, AKT, and glycogen synthase kinase $3 \beta$ (GSK3 $\beta$ ) associated with cardiac hypertrophy and further inhibit the overexpression of the low downstream factor GATA4 in the nucleus. Therefore, this study supported that Xinmailong Injection can prevent myocardial decompensated hypertrophy from its inhibition of phosphorylation of ERK1/2, AKT/GSK $3 \beta$ pathway, and inhibition of overexpression of GATA4.

3.3.6. Protective Mechanism of Xinmailong Injection on Vascular Structure and Function. Vascular endothelial cells are located between plasma and vascular tissue. It not only completes the exchange of metabolites of plasma and tissue fluids but also synthesizes and secretes a variety of biologically active substances. Bioactive substances can maintain the normal contraction and relaxation of blood vessels, regulate blood pressure (BP), and balance blood coagulation and hemolysis.

Endothelin (ET) and nitric oxide (NO) are a major endothelium-dependent relaxation and contraction vascular substance. ET has the functions of contracting blood vessels, promoting platelet adhesion and aggregation, and damaging the vascular endothelium. NO has the functions of dilating blood vessels, inhibiting blood cell adhesion, and anti-vascular smooth muscle cell proliferation. Wu Jianxin et al. [25] observed the effect of Xinmailong Injection on pulmonary hypertension induced by MCT and found that
Xinmailong Injection can significantly reduce pulmonary artery pressure (PAP) and increase plasma NO level and ET level. This indicates that Xinmailong Injection can reduce pulmonary hypertension by regulating vascular endothelial factor.

The expression of endothelin-1 (ET-1) and myocardial hypoxia-inducible factor- $1 \alpha$ (HIF- $1 \alpha$ ) can reflect the hypoxic state of the body. Huang Lixin and Wu Xingheng [26] found that the expression of HIF- $1 \alpha$, ET-1, and CK was significantly increased in neonatal rats with asphyxia, and the expression of HIF- $1 \alpha$ was positively correlated with plasma ET-1 level $(r=$ $0.876, \mathrm{P}<0.01)$. Xinmailong Injection can effectively inhibit the expression of HIF-1 $\alpha$ in the myocardium and decrease the levels of ET-1 and CK in plasma. This indicates that Xinmailong Injection can improve the myocardial ischemia and hypoxia injury by regulating vascular endothelial factor, making the blood vessels patency and blood flow normal, so as to ensure sufficient blood and oxygen supply to the myocardium.

Prostacyclin (PGI2) is an important vasodilator secreted by vascular endothelial cells, and its stable metabolite is 6keto-prostaglandin F1a (6-Keto-PGFla). By observing the intervention effect of Xinmailong Injection on vascular endothelial cells in rats with arteriosclerosis, Zhang Wenjing et al. [27] found that Xinmailong Injection can significantly reduce plasma ET levels and increase serum NO levels and plasma 6-Keto-PGFla levels. In addition, Sun Lin and Zhang Wenjing [28] also found that Xinmailong Injection can significantly reduce total cholesterol (TC), triglyceride (TG), and low-density lipoprotein cholesterol (LDL-C) levels and raise high-density lipoprotein cholesterol (HDL-C) level in the serum of atherosclerotic (AS) rats. It is suggested that Xinmailong Injection can prevent and delay the progression of AS by regulating vascular endothelial factor and improving blood lipid status.

3.3.7. Mechanism of Antiarrhythmia of Xinmailong Injection. Wu Jianxin et al. [29] found that pretreatment of healthy rabbits with an Injection of Xinmailong Injection through the ear vein can significantly prolong the survival time of rabbits during continuous perfusion of $\mathrm{BaCl}_{2}$ solution and reduce the arrhythmia caused by a bolus injection of $\mathrm{BaCl}_{2}$ solution. For rabbit models with arrhythmia, Xinmailong Injection can significantly improve the rate of sinus arrhythmia conversion. Arrhythmias such as atrial fibrillation or rapid atrial stimulation can cause a shortening of the atrial effective refractory period (AERP) [44]. Li Fengde et al. [44] found that continuous injection of 5day Xinmailong Injection for rabbits can effectively prevent the shortening of AERP200 and AERP150 caused by rapid pacing for 6 hours. This indicates that Xinmailong Injection can effectively prevent atrial electrical remodeling. Li Hui et al. [20] found that Xinmailong Injection can improve the prolongation of QT and QTc on electrocardiogram induced by epirubicin. In addition, prolongation of QT dispersion (QTd) in patients with ischemic heart disease can predict the occurrence of malignant arrhythmia and sudden death. Clinical researches found that Xinmailong Injection can 
reduce the prolongation of QTd and prevent the occurrence of arrhythmia.

The researchers considered that the antiarrhythmia function of Xinmailong Injection may be related to the following mechanisms: (1) by regulating the activity of channel proteins transporting $\mathrm{Na}^{+}, \mathrm{Ca}^{2+}$, and $\mathrm{K}^{+}$on the cardiomyocyte membrane to ensure the normal cardiac electrical conduction pathway and (2) by improving myocardial cell metabolism and scavenging oxygen free radicals to protect the integrity of myocardial cell membrane structure and function, thereby maintaining the stability of a variety of ion channels. At present, the specific mechanism of antiarrhythmia of Xinmailong Injection is still unclear and needs further exploration [45].

\section{Discussion}

4.1. Intervention Mechanism of Xinmailong Injection on Cardiovascular Disease. This study shows that the intervention mechanism of Xinmailong Injection on cardiovascular disease mainly includes the following points: (1) Xinmailong Injection inhibits OS by inhibiting the synthesis of ROS and promoting the activity of antioxidant enzymes and reduces the expression of proinflammatory factors by inhibiting the activation of NF-kB system. Therefore, the damage, degeneration, and necrosis of cardiomyocytes are alleviated. (2) Xinmailong Injection protects cardiomyocytes from drug toxicity by activating endogenous ROS clearance systems and regulating cellular autophagy. (3) Xinmailong Injection enhances myocardial contractility by activating ECC excitability and promoting $\mathrm{Ca}^{2+}$ influx. Maintaining the normal transport of $\mathrm{Na}^{+}, \mathrm{Ca}^{2+}$, and $\mathrm{K}^{+}$on the myocardial cell membrane may also be an important mechanism of antiarrhythmia of Xinmailong Injection. (4) Xinmailong Injection regulates the dynamic balance of MMPs and TIMPs by inhibiting the overexpression of TGF- $\beta 1$ and CTGF, thereby maintaining the normal structure and function of ECM, inhibiting collagen deposition and MF. (5) Xinmailong Injection delays the development of cardiac hypertrophy by inhibiting the phosphorylation of extracellular regulated protein kinases $1 / 2$, AKT, and GSK $3 \beta$ proteins and overexpression of the downstream factor GATA4 in the nucleus. (6) Xinmailong Injection protects the structure and function of blood vessels by regulating vascular endothelial factor disorder and improving blood lipid status so that the heart muscle can obtain sufficient blood and oxygen supply. (7) The mechanism of antiarrhythmia of Xinmailong Injection is not clear. This may be related to its regulation of the activity of ion transport pathway proteins on the cell membrane to maintain the normal transport of $\mathrm{Na}^{+}, \mathrm{Ca}^{2+}$, and $\mathrm{K}^{+}$on the myocardial cell membrane. This may also be related to its ability to scavenge oxygen free radicals to protect cell membrane structure and function.

Excessive activation of the neuroendocrine system is considered to be a key process leading to HF. Among them, the renin-angiotensin-aldosterone system (RAAS) plays a major role and can affect multiple links of the above mechanisms. Clinical studies have found that Xinmailong Injection can inhibit the excitability of the RAAS system. This may be a key intervention mechanism of Xinmailong Injection on cardiovascular disease. It is expected to carry out a large number of relevant basic researches for further exploration.

\subsection{Clinical Application Value of Xinmailong Injection in the} Treatment of HF. The current conventional drugs for treating HF have certain deficiencies while improving symptoms. Diuretics can cause electrolyte imbalance and hypotension. Beta blockers have a significant negative inotropic effect on the heart and may cause bradycardia or conduction block. Digitalis drugs may cause poisoning due to the narrow treatment width. Meta-analyses [46-48] showed that Xinmailong Injection can significantly improve a variety of clinical indicators related to cardiac function, thereby improving the clinical efficacy of patients with HF. In addition, Xinmailong Injection had the effects of regulating BP, enhancing myocardial contractility, and preventing arrhythmia and thus can prevent the occurrence of the above adverse reactions to a certain extent. However, Xinmailong Injection combined with conventional treatment of western medicine may cause adverse skin reactions $[R R=2.04,95 \%$ CI $(1.05$, 3.96), $\mathrm{P}=0.03$ ] [49]. In addition, the clinical cost-effectiveness analysis of Xinmailong Injection in the treatment of $\mathrm{HF}$ showed that, compared with the conventional treatment group, the combination of Xinmailong and conventional treatment group had low cost, high efficiency, and better economic benefits. In summary, Xinmailong Injection plays an important role in improving the quality of life of patients and reducing the medical burden on families and society. Its medicinal value has great potential for development.

4.3. The Direction and Thinking of the Future Research of Xinmailong Injection. Xinmailong Injection is a multicomponent pharmaceutical preparation whose active ingredients can act on multiple targets of the body through different routes. Clinical studies have shown that Xinmailong Injection can effectively treat a variety of cardiovascular system related diseases, such as pulmonary heart disease (PHD) [50, 51], ischemic cardiomyopathy (IHD) [52], dilated cardiomyopathy (DCM) [53], cardiogenic shock [54], and heart and kidney syndrome $[55,56]$, etc. Due to the lack of basic research in related fields, the intervention mechanism of Xinmailong Injection is not yet clear. More and more tissue cytology research, molecular biology research, and genetic research are being expected. To study the pharmacological mechanism of Xinmailong Injection, it is necessary to study not only the single active ingredients but also the common pathways, regulatory factors, and targets of the active ingredients in the process of initiation, so as to construct a complete system of intervention mechanisms. This will provide a reference for the formulation of more reasonable clinical prescriptions for Xinmailong Injection and further research and development of new drugs. 


\section{Conclusion}

Xinmailong Injection can protect cardiomyocytes and maintain the normal function of the heart in various ways, thus effectively preventing the development of cardiovascular disease. Therefore, Xinmailong Injection has great potential for clinical application, and more basic researches need to be carried out to explore the medicinal value of Xinmailong Injection.

\section{Conflicts of Interest}

The authors declare that there are no conflicts of interest.

\section{Authors' Contributions}

Shan-Shan Lin and Chun-Xiang Liu contributed equally to this work. They took part in the design of the study, performed the literature survey, and drafted the manuscript. Xian-Liang Wang and Jing-Yuan Mao took part in the design and implementation of the study. All authors have read and approved the final manuscript.

\section{Acknowledgments}

This work was supported by the Tianjin Enterprise Science and Technology Commissioner Project (NO. 18JCTPJC64900), the National Natural Science Foundation of China (NO. 81804218), the Ministry of Education of People's Republic of China "Program for Innovative Research Team in University” (NO. IRT_16R54), and the Tianjin Science and Technology Program (NO. 15ZXLCSY00020).

\section{References}

[1] W. W. Chen, R. L. Gao, L. S. Liu et al., "Summary of China cardiovascular disease report 2017," Chinese Circulation Journal, vol. 30, no. 1, pp. 1-8, 2018.

[2] H. X. Man, L. Huang, K. G. Na, Q. Y. Tan, Y. S. Yang, and P. Y. Xiao, "Research progress of chemical component and biological activity in medicinal Periplaneta Americana," AntiInfection Pharmacy, vol. 11, no. 5, pp. 403-407, 2014.

[3] J. Y. Qi, J. Yu, Y. F. Tan et al., "Mechanisms of Chinese medicine Xinmailong's protection against heart failure in pressure-overloaded mice and cultured cardiomyocytes," Scientific Reports, vol. 7, p. 42843, 2017.

[4] K. J. Chen, Z. G. Wu, M. J. Zhu, J. Y. Mao, and H. Xu, "Expert consensus on diagnosis and treatment of chronic heart failure by integrative Chinese and western medicine," Chinese Journal of Integrated Traditional Chinese and Western Medicine, vol. 36, no. 2, pp. 133-141, 2016.

[5] Dali Medical College, Xinmailong extract and Xinmailong pharmaceutical preparation, China, 1996.

[6] Doctor Society of Integrative Medicine, Chinese Medical Doctor Association, and ETAL, "Guidelines for the diagnosis and treatment of acute myocardial infarction by integrative Chinese and western medicine," Chinese Journal of Integrated Traditional Chinese and Western Medicine, vol. 38, no. 3, pp. 272-284, 2018.

[7] B. Yao, X. He, Y. Lin, and W. Dai, "Cardioprotective effects of anisodamine against myocardial ischemia/reperfusion injury through the inhibition of oxidative stress, inflammation and apoptosis," Molecular Medicine Reports, vol. 17, no. 1, pp. 12531260, 2018.

[8] A. M. Al-Taweel, M. Raish, S. Perveen et al., "Nepeta deflersiana attenuates isoproterenol-induced myocardial injuries in rats: possible involvement of oxidative stress, apoptosis, inflammation through nuclear factor (NF)- $\kappa \mathrm{B}$ downregulation," Phytomedicine, vol. 34, pp. 67-75, 2017.

[9] J. Qu, N. Lin, and Q. L. Ding, "ROS-induced NRP3 inflammasome activation and cardiovascular diseases," Pharmacy Today, vol. 27, no. 12, pp. 855-858, 2017.

[10] J. X. Wu, Y. G. Zhou, and R. X. Niu, "Protective effect of Xinmailong on myocardial injury induced by isoproterenol in rabbits," Journal of Dali Medical College, vol. 4, no. 1, pp. 5-8, 1995.

[11] J. X. Wu, Y. X. Wang, R. X. Niu, Y. G. Zhou, and N. Liang, "Protective effect of Xinmailong on isoproterenol-induced ischemic myocardium in rats," Chinese Journal of Pathophysiology, vol. 18, no. 1, pp. 97-98, 2002.

[12] K. L. Tian and Z. H. Yang, "Protective effect of Xinmailong on rabbits with ischemia-reperfusion injury," Chinese Journal of Ethnomedicine and Ethnopharmacy, vol. 19, no. 12, pp. 22-25, 2010.

[13] Z. Li, S. Li, L. Hu et al., "Mechanisms underlying action of xinmailong injection, a traditional chinese medicine in cardiac function improvement," African journal of traditional, complementary, and alternative medicines: AJTCAM, vol. 14, no. 2, pp. 241-252, 2017.

[14] H. X. Cao, R. M. Zhong, and X. H. Wu, "The protection of Xinmailong against reperfusion injury in immature rabbits myocardial ischemia," Journal of Jiangxi Medical College, vol. 49, no. 10, pp. 15-18, 2009.

[15] L. J. Zhang, R. M. Zhong, and X. H. Wu, "Effects of Xinmailong on expression of NF- $\kappa \mathrm{B}$ and TNF-a in myocardium of asphyxiated neonatal rats," Journal of Nanchang University (Medical Science), vol. 51, no. 3, pp. 14-17, 2011.

[16] W. Liu, X. B. Duan, and X. Xu, "Preventive effect and mechanism of Xinmailong Injection on cardiotoxicity induced by adriamycin in rats," Chinese Journal of Integrative Medicine on Cardio-/Cerebrovascular Disease, vol. 12, no. 2, pp. 210-211, 2014.

[17] W. Liu, Y. H. Wang, and Q. Wang, "Preventive effect and mechanism of Xinmailong Injection on cardiotoxicity induced by adriamycin in rats," Technology Wind, vol. 1, no. 29, 2016.

[18] X. B. Duan, W. Liu, and Y. F. Wang, "Experimental study of Xinmailong Injection on cardiotoxicity induced by epirubicin in rats," Chinese Journal of Integrative Medicine on Cardio/Cerebrovascular Disease, vol. 16, no. 8, pp. 1038-1040, 2018.

[19] L. L. Yuan and Y. Jiang, "Protective mechanism of Xinmailong Injection on adriamycin-induced cardiomyocyte injury," Chinese Journal of Integrative Medicine on Cardio/Cerebrovascular Disease, vol. 15, no. 23, pp. 2977-2980, 2017.

[20] H. Li, Y. Mao, Q. Zhang et al., "Xinmailong mitigated epirubicin-induced cardiotoxicity via inhibiting autophagy," Journal of Ethnopharmacology, vol. 192, pp. 459-470, 2016.

[21] J. Li, W. Shi, J. Zhang, and L. Ren, "To explore the protective mechanism of pten-induced kinase 1 (PINK1)/parkin mitophagy-mediated extract of periplaneta americana on lipopolysaccharide-induced cardiomyocyte injury," Medical Science Monitor, vol. 25, pp. 1383-1391, 2019.

[22] F. Peng, C. S. Fang, and X. B. Liu, "Effect of Xinmailong Injection on free calcium content in rat myocardial cells," 
Traditional Chinese Drug Research and Clinical Pharmacology, vol. 13, no. 4, pp. 224-269, 2002.

[23] F. Peng, X. B. Liu, C. S. Fang, and Z. K. Yang, "Effect of Xinmailong Injection on cytosolic free calcium and lipid peroxidation in rat heart cells after anoxia and reoxygenation damage," Journal of Chinese Pharmaceutical Sciences, vol. 12, no. 1, pp. 33-35, 2003.

[24] G. H. Liu, J. Huang, and Y. S. Liu, "Effect of Xinmailong Injection on myocardial fibrosis in rats with alcoholic cardiomyopathy," Chinese Journal of Integrative Medicine on Cardio/Cerebrovascular Disease, vol. 15, no. 10, pp. 1177-1180, 2017.

[25] G. H. Liu, J. Huang, and Y. S. Liu, "Experimental study of the XML injection antagonizing rat pulmonary hypertension by monocrotaline," Journal of Medical Research, vol. 38, no. 1, pp. 29-32, 2009.

[26] L. X. Huang and X. H. Wu, "Effect of Xinmailong on hypoxiainducible factor- $1 \alpha$ expression in neonatal rats with asphyxia," Chinese Journal of Contemporary Pediatrics, vol. 11, no. 8, pp. 683-686, 2009.

[27] W. J. Zhang, L. L. Wang, and S. Y. Song, "Protective effect of Xinmailong infection vascular endothelial cells of atherosclerosis rats," Journal of Hebei North University (Natural Science Edition), vol. 28, no. 2, pp. 79-81, 2012.

[28] L. Sun and W. J. Zhang, "Effect of Xinmailong (XML) Injection on blood lipids in rats with atherosclerosis," Journal of Hebei North University (Natural Science Edition), vol. 28, no. 1, pp. 6769, 2012.

[29] J. X. Wu, R. X. Niu, Y. L. Huang, Y. Qin, and X. Q. Huang, "Prevention and treatment effect of Xinmailong on experimental arrhythmias in rabbits," Medical Journal of Dali College, vol. 11, no. 2, pp. 18-19, 2002.

[30] J. X. Wu, R. X. Niu, Y. L. Huang, Y. Qin, and X. Q. Huang, "Effect of Xinmailong Injection on atrial electrical remodeling in rabbits with rapid atrial pacing," Chinese Journal of Integrative Medicine on Cardio/Cerebrovascular Disease, vol. 16, no. 17, pp. 2489-2491, 2018.

[31] G. Filomeni, D. de Zio, and F. Cecconi, "Oxidative stress and autophagy: the clash between damage and metabolic needs," Cell Death \& Differentiation, vol. 22, no. 3, pp. 377-388, 2015.

[32] S. Yu and Y. Q. Ni, "Autophagy, oxidative stress and nephropathy," Progress Physiological Sciences, vol. 49, no. 4, pp. 275-279, 2018.

[33] M. Gyöngyösi, J. Winkler, I. Ramos et al., "Myocardial fibrosis: biomedical research from bench to bedside," European Journal of Heart Failure, vol. 19, no. 2, pp. 177-191, 2017.

[34] R. D. Brown, S. K. Ambler, M. D. Mitchell, and C. S. Long, "The cardiac fibroblast: therapeutic target in myocardial remodeling and failure," Annual Review of Pharmacology and Toxicology, vol. 45, pp. 657-687, 2005.

[35] R. Martos, J. Baugh, M. Ledwidge et al., "Diastolic heart failure: evidence of increased myocardial collagen turnover linked to diastolic dysfunction," Circulation, vol. 115, no. 7, pp. 888-895, 2007.

[36] K. Y. Goh, L. He, J. Song et al., "Mitoquinone ameliorates pressure overload-induced cardiac fibrosis and left ventricular dysfunction in mice," Redox Biology, vol. 21, pp. 1-15, 2019.

[37] W. J. Guang, B. F. Ao, W. W. Ouyang, S. F. Su, and B. Lu, "Effects of TGF- 11 on myocardial fibrosis due to different reasons," Science \& Technology Review, vol. 34, no. 2, pp. 221-225, 2016.

[38] S. Fu, Y. L. Li, Y. T. Wu, Y. Yue, and Y. Gao, "Icariside 2 ameliorates myocardial fibrosis in spontaneously hypertensive rats through regulating expression of MMP-2, MMP-9, and TIMP-1," Science Technology Review, vol. 34, no. 9, pp. 1253-1257, 2018.

[39] C. M. Zhao, Q. F. Song, X. Z. Wang et al., "Effect of Xinmailong on serum matrix metalloproteinase-1 in patients with congestive heart failure," Chinese Journal of Gerontology, vol. 34, no. 12, pp. 3455-3456, 2014.

[40] C. M. Zhao, Q. F. Song, X. Z. Wang et al., "The effect of Xinmailong on matrix metalloproteases- 9 in patients with congestive heart failure (CHF)," Chinese Journal of Coal Industry Medicine, vol. 17, no. 2, pp. 173-176, 2014.

[41] A. Leask, "Potential therapeutic targets for cardiac fibrosis: TGF $\beta$, angiotensin, endothelin, CCN2, and PDGF, partners in fibroblast activation," Circulation Research, vol. 106, no. 11, pp. 1675-1680, 2010.

[42] Y. E. Koshman, N. Patel, M. Chu et al., "Regulation of connective tissue growth factor gene expression and fibrosis in human heart failure," Journal of Cardiac Failure, vol. 19, no. 4, pp. 283294, 2013.

[43] Q. Liang, L. J. de Windt, S. A. Witt, T. R. Kimball, B. E. Markham, and J. D. Molkentin, "The transcription factors GATA4 and GATA6 regulate cardiomyocyte hypertrophy in vitro and in vivo," The Journal of Biological Chemistry, vol. 276, no. 32, pp. 30245-30253, 2001.

[44] M. C. E. F. Wijffels, C. J. H. J. Kirchhof, R. Dorland, and M. A. Allessie, "Atrial fibrillation begets atrial fibrillation: a study in awake chronically instrumented goats," Circulation, vol. 92, no. 7, pp. 1954-1968, 1995.

[45] H. Tang, J. Li, Z. H. Huang, Z. Q. Ren, and J. P. Ning, "Effect of Xinmailong on QT dispersion and clinical efficacy in patients with ischemic heart failure," Chinese Medical Journal, vol. 8, no. 9, pp. 1211-1213, 2013.

[46] J. M. Zhang, Y. D. Shang, X. R. Wu, Y. J. Fu, and C. Y. Xie, "Clinical efficacy of Xinmailong Injection in the treatment of chronic heart failure: a meta-analysis," Medica of china family physicians, vol. 17, no. 12, pp. 1388-1393, 2014.

[47] Y. D. Zuo, X. C. Liu, and M. Li, "A meta-analysis of Xinmailong Injection on treating chronic heart failure," Clinical Journal of Chinese Medicine, vol. 7, no. 2, pp. 11-14, 2015.

[48] X. H. Lu, L. Zhang, J. B. Wang et al., "Clinical efficacy and safety of Xinmailong Injection for the treatment of chronic heart failure: a meta-analysis," Frontiers in Pharmacology, vol. 9, pp. 1-14, 2018.

[49] Z. Q. Liu, H. B. Liu, and B. L. Wang, "Safety of Xinmailong Injection in the treatment of heart failure:a meta-analysis," Journal of China Pharmacy, vol. 29, no. 22, pp. 3152-3157, 2018.

[50] G. H. Liu, H. R. Shi, and J. Huang, "Therapeutic effect of Xinmailong Injection on chronic pulmonary heart disease," World Latest Medicine Information, vol. 18, no. 31, pp. 132-136, 2018.

[51] G. H. Liu, H. R. Shi, and J. Huang, "Effect of Xinmailong Injection on blood gas analysis, D-dimer and hemoglobin in patients with chronic pulmonary heart disease," World Latest Medicine Information, vol. 18, no. 33, pp. 141-143, 2018.

[52] G. F. Song, F. Shao, and L. Wang, "Therapeutic effect of Xinmailong Injection on patients with ischemic cardiomyopathy," Chinese Journal of Practical Medicine, vol. 11, no. 31, pp. 115-116, 2016.

[53] W. Q. Liu, Q. Ao, X. W. Wang, and X. H. Yin, "Effect of Xinmailong Injection on brain natriuretic peptide and high-sensitive C-reactive protein in patients with dilated cardiomyogenic," Contemporary Medicine, vol. 21, no. 18, pp. 134-135, 2015. 
[54] Z. R. Ge, L. Li, and S. B. Jiang, "Clinical observation on Xinmailong Injection and IABP in the treatment of acute myocardial infarction complicated with cardiac shock," Chinese Journal of Integrative Medicine on Cardio/Cerebrovascular Disease, vol. 16, no. 4, pp. 434-436, 2018.

[55] P. Sui, "Clinical curative effect analysis of Xinmailong Infection on I type cardiac-renal syndrome," Guide of China Medicine, vol. 14, no. 24, pp. 12-13, 2016 (Chinese).

[56] W. H. Shao, S. X. Wang, C. X. Lv, F. Li, X. Q. Li, and N. Wang, "Effect of Xinmailong Injection on NGAL, hs-cTnT, and RAAS in elderly patients with type 2 cardio-renal syndrome," The Journal of Practical Medicine, vol. 35, no. 4, pp. 654-657, 2019. 


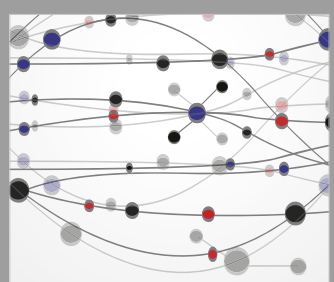

The Scientific World Journal
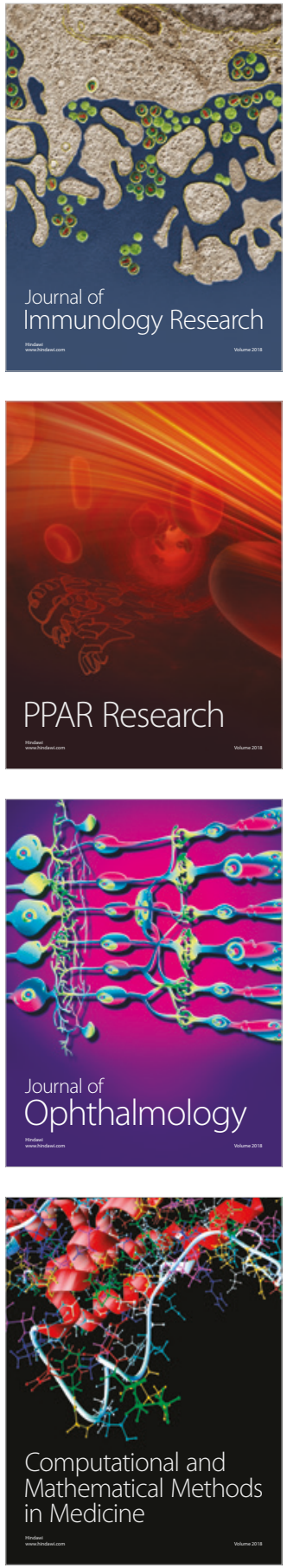

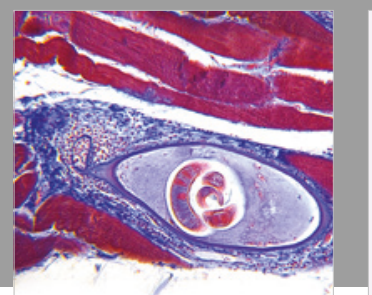

Gastroenterology Research and Practice

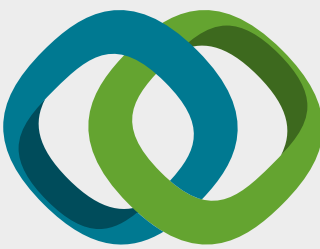

\section{Hindawi}

Submit your manuscripts at

www.hindawi.com
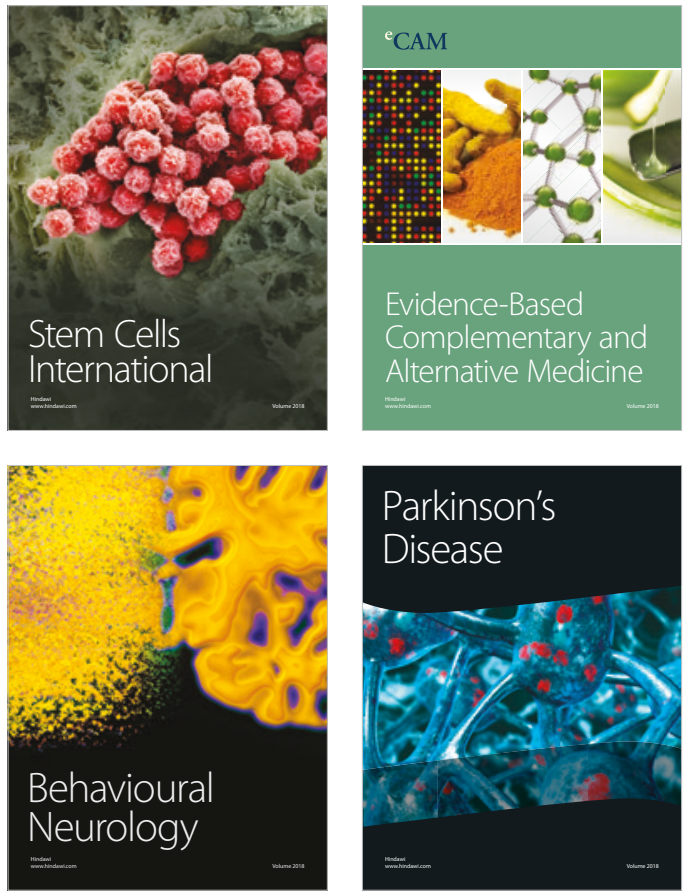

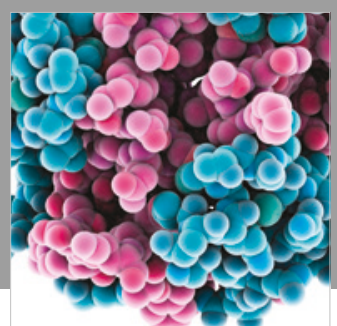

ournal of

Diabetes Research

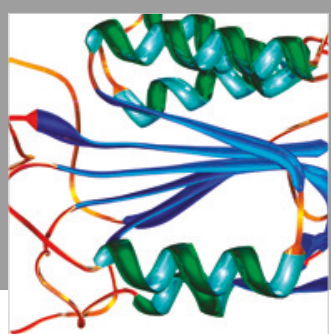

Disease Markers
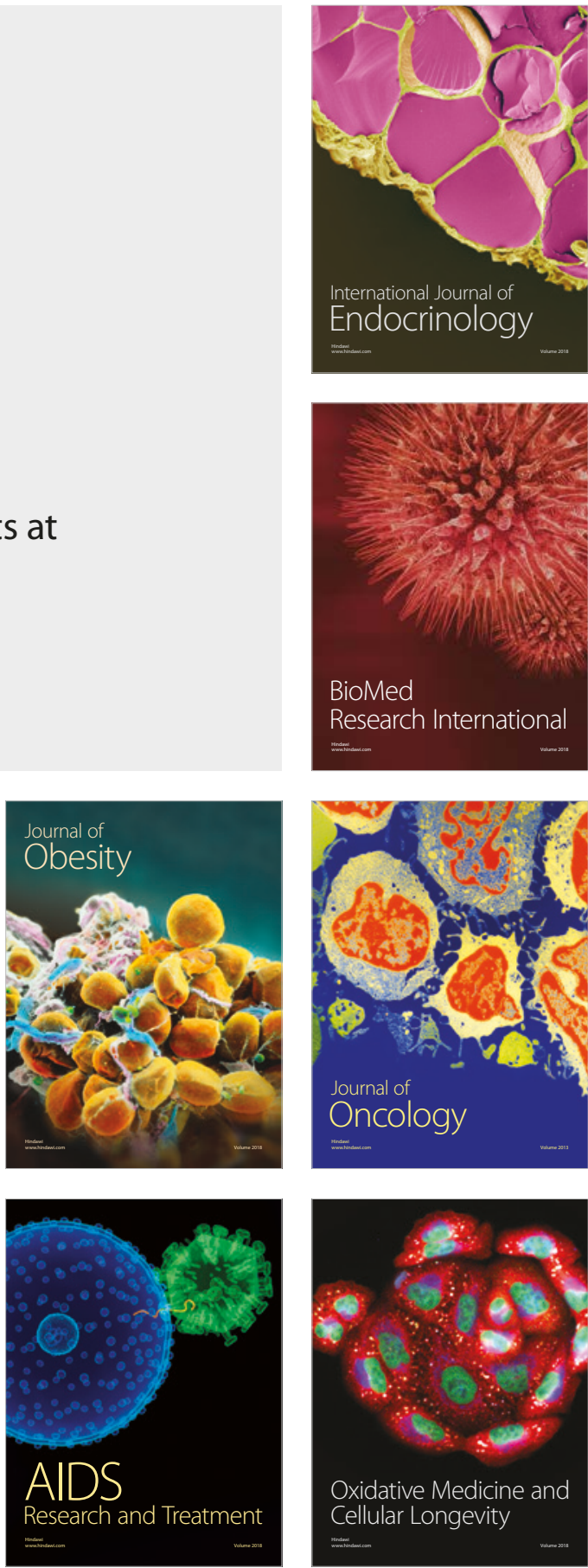